\title{
The Application of Blockchain-Based Life Cycle Assessment on an Industrial Supply Chain
}

\author{
Xuda Lin ${ }^{1,2,}$, Xing Li ${ }^{3}$, Sameer Kulkarni ${ }^{4}$, and Fu Zhao ${ }^{1,4}$ \\ 1 Division of Environmental and Ecological Engineering, Purdue University, West Lafayette, 47907, US \\ 2 Department of Statistics, Purdue University, West Lafayette, 47907, US \\ 3 School of Civil Engineering, Purdue University, West Lafayette, 47907, US \\ 4 School of Mechanical Engineering, Purdue University, West Lafayette, 47907, US; \\ lin468@purdue.edu (Lin, X.); li1856@purdue.edu (Li, X.); kulkar15@purdue.edu (Kulkarni, S.); \\ fzhao@purdue.edu (Zhao, F.) \\ * Correspondence: lin468@purdue.edu; Tel.: +1-765-(409)-6349
}

\begin{abstract}
Life Cycle Assessment (LCA) is a widely recognized tool used to evaluate environmental impacts of a product or process, based on the environmental inventory database and bills of material. Data quality is one of the most significant factors affecting the analysis results. However, currently most datasets in inventory databases are generic i.e., they may represent material and energy flow of a process at market average, instead of a specific process used by a manufacturer. As a result, stockholders are unable to track their supply chain to find out the actual environmental impact from each supplier and to compare the environmental performance of alternative options. In this paper, we developed a new framework i.e., blockchain based LCA (BC-LCA), where block-chain technology is adapted to secure and transmit inventory data from upstream suppliers to downstream manufacturers. With BC-LCA, more specific data can be acquired along the supply chain in a real-time manner. Moreover, the availability, accuracy, privacy, and automatic update of inventory data can be improved. A case study is provided based on an industrial supply chain, to demonstrate the utilization of BC-LCA.
\end{abstract}

Keywords: Life Cycle Assessment; Blockchain; Supply Chain

\section{Introduction}

In recent decades, environmental problems have been an increasingly global issue, affecting everyone. Typical environmental problems include global warming, chemical pollution, depletion of nature resources and the loss of biodiversity [1]. The blooming of new technologies and demand of products brings growth of industry, but also leads to more serious environmental problems. Thus, to produce a more accurate and fast quantification of the environmental impacts caused by a certain process, a reliable tool is required, which could measure and calculate the environmental impacts in different aspects [2].

Life Cycle Assessment (LCA) is a widely used tool to analyze environmental impacts. The users of LCA include governments, non-governmental organizations, industrial sectors, and academic and education institutes. The results of LCA could provide customers a reference, so that they can make comparisons. LCA can also benefit decision making, by providing information on several alternative products or processes, so that there could be some space for trading off [3]. 


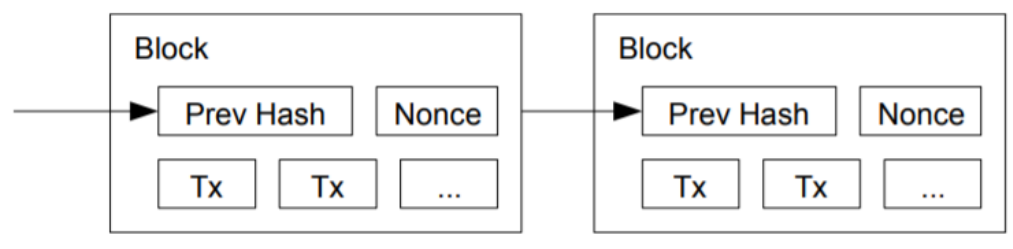

Figure 1. Classic blockchain framework in Bitcoin [4].

Blockchain, prominently implemented in cryptocurrency Bitcoin [4], could be a promising and complementary addition to LCA. Besides cryptocurrency, blockchain is widely used in financial service, health and medical service, energy rebalancing, agricultural service and so on. Previous research shows that blockchain can also be applied to improve the sustainable performance as well as the resilience of supply chain [5-6]. LCA has a very close relationship with the bill of material for products, which are derived from the supply chain. Thus, the integration of blockchain and LCA theoretically and practically would lead to a more convenient and reliable database.

Blockchain is a data structure, composed as a chain of blocks interconnected in a unidirectional order via an encrypted Hash function. The classic blockchain framework is shown in Figure 1. Each block contains three parts: a previous hash, a nonce and transaction data. The previous hash contains information acting as a pointer to the previous block, allowing us to back trace the information along the blockchain. The second part is a nonce, which formats the hash of the whole block into a string with a certain number of zeros in the very first several digits. The third part is transaction data, which would be stored in a tree [7].

In classic blockchain, mining is a process performed to find a nonce such that it can make the hash of the whole block into a string with a certain number of zeros in the very first several digits. Each node must generate a nonce, then do the SHA-256 (an encipher algorithm), and repeat, until such a nonce is found. This circular process is defined as proof -of-work, as each processor (CPU) can only finish a limited number of SHA calculations. After the nonce is found, the node can pack up the transaction data into a block, using this nonce, and broadcast this block to the whole network, allowing all the nodes in the network to verify if this block is a legal (which means this block follows all the rules and restrictions on blockchain) one. After the verification, all other nodes would copy the information on that block, so that all the transaction data on that block could be applied. The node who finds out that nonce and pack up the transaction data would be rewarded several cryptocurrencies (e.g., Bitcoin or Ethereum).

The research about using blockchain on life cycle assessment is in its early stages. Smetana mentions the impact of artificial neural network (ANN) and blockchain on revolutionizing Material Flow Analysis (MFAs) and LCAs [8], which is more like a conceptual reference, saying that some new technology, especially ANN and blockchain, could provide positive improvement on MFAs and LCAs. In 2020, more research about blockchain-related life cycle assessment was published. A framework about implementation of blockchain-based LCAs was developed [9], with a budget estimation. This study combined blockchain-based LCA with Internet of Things (IoT), trying automatically fetch data from sensors. A fuzzy DEMATEL analysis on blockchain-based LCA in China was provided [10], which gives a result that blockchain-based LCA could improve manufacture data accuracy. A strategy related research on blockchain-enabled LCA was published [11], discussing the concern that BC-LCA might get support from strategy. In 2021, a conference paper of blockchain-based LCA and aircraft related application was published in CIRP (The international academy of production engineering) [12], which provides a good example on BC-LCA application.

This paper is organized as follows. In Section 2, the disadvantages of current LCA framework that can be fixed by a blockchain enabled LCA (BC-LCA) would be discussed. Section 3 presents the key assumptions that this study based on. Section 4 provides the 
framework and mechanism about BC-LCA, and its benefits would be discussed in Section 5. A case study discussing the potential implementation of BC-LCA for a chemical generation process is also provided in Section 6.

\section{Disadvantages on Current LCA}

In any product's supply chain, there are many enterprises (suppliers, shipping, manufacturing, raw material acquisition, etc..), which considered as "nodes". The partnership in-between two nodes, is an "edge" [13]. LCA often faces challenges in collecting reliable data from supply chain, especially from a supply chain with multiple nodes and complex edges. Theoretically, the results of LCA only depend on two critical parts: the bills of material and environmental inventory data. Obtaining reliable data is a very challenging problem [14]. In classic LCA, each node should obtain the environmental inventory data by its own. Currently, most facilities would use published environmental inventory databases such as ecoinvent. All the data provided by researchers are under some assumptions (e.g., the scope definition), but when these data are implied, these assumptions might be ignored by the user. In fact, not all the LCA research can find appropriate data. The practical LCA application needs the database that has higher accuracy, higher availability and is more specific to the product or process being studied.

\subsection{Inefficiency of Data Transmission}

The most popular environmental inventory databases are always from centralized companies and organizations (e.g., Sphera, ecoinvent). These databases are collected and compiled by a specific organization first, and then published to users all over the world. Theoretically, all the data transmission in centralized structure should go through a center. This center could be a facility (e.g., a bank in wired transfer process) or a network (e.g., cellular network in the process of a phone call) and should process all the restrictions and the verifications. It might be too abstract to directly talk about the data transmission efficiency without any example. Banking system is one of the most typical centralized structures, and it is close to daily life, easy to explain. If customer A wants to transfer 3 dollars to customer B, the bank needs to check if there's enough money on A's account, and whether the account information about B is correct. All the restrictions and verifications happen in the bank, which is easy to update the rule. However, wired money transfer may take up to days, and international money transfer may take up to weeks. This is a tradeoff for the efficiency in using centralized structures. On the one hand, considering restriction and verification processes, centralized structure is efficient enough and powerful, because all the restrictions and verifications are easy to imply [15]; on the other hand, there is a significant disadvantage commonly shared by centralized structures: data transmission inefficiency [16]. 


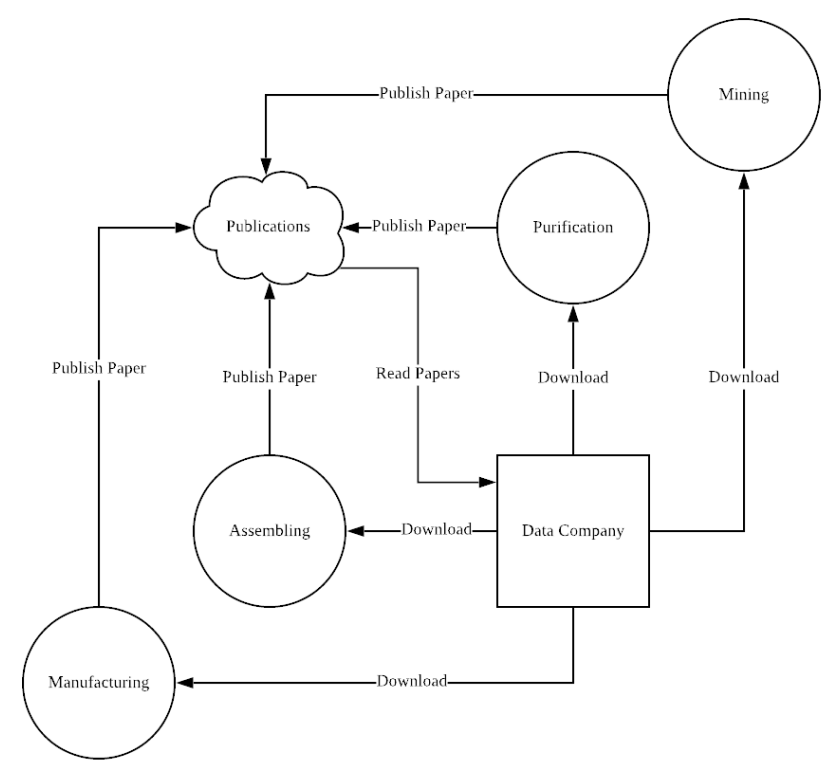

Figure 2. Centralized LCA Structure.

The inefficiency of centralized structure in data transmission is worse in LCA data exchange, as there is no such a shared centralized platform to do the data collection. The procedure of data updating in LCA is much more inefficient: If researcher A have done some LCA research and updated some data, researcher A must publish a paper to describe this update. Then, the environmental inventory data companies and organizations (e.g., PRé, ecoinvent, Sphera) must keep reading papers to catch up with this kind of updates, so that the database companies and organizations can then record these updates into a new version of environmental inventory database. Eventually, all the users could see this update after the new version is released by the database companies and organizations. Figure 2 shows the whole process based on the previous example: if any node in a supply chain wants to do LCA, it is necessary to download the environmental inventory data from a centralized data company. Meanwhile, the LCA results would be published via papers or other publications, which could be used as data source for these companies or organizations who publish environmental inventory database.

\subsection{Lack of Data Availability}

Current environmental inventory database contains a lot of information about different kinds of manufacturing processes. The recent ecoinvent database contains more than 16,000 datasets [17]. For researchers, if generic environmental inventory information is needed about one generic type of process, then the aggregated (average) environmental inventory dataset might be enough. For example, if researchers are interested in the comparison between two different types of products, such as a paper cup and a polymer cup, then the generic data might work. However, if a user wants to know the environmental impact of one specific process, then generic information is not enough. For example, if a user wants to know the environmental performance about a laptop with specific model, such as HP Envy X360 with 8GB RAM from Kingston, 1T HDD from Western Digital and Core i5-8500 processor from Intel, then generic data can only provide the user the environmental impact about a generic laptop, instead of this specific model. With current database, a user will never know the accurate environmental impact of one specific product or process.

\subsection{Concerns on Data Privacy}

According to ISO 14044, the results of LCA depend on two critical parts: environmental inventory database and bills of material. In most cases, environmental inventory data are provided by public organizations or companies, which is a typical centralized 
structure. The reason behind is, manufacturers are hesitant to share their data, no matter if it is their inventory data or environmental inventory data of their final products. Even within one company, the data privacy problem also can exist between different departments. The priority of protecting their own data is much higher than sharing these data to benefit the whole supply chain. Thus, probably the only way to solve this problem is finding a trustworthy third party (e.g., a data company) to manage these data, and make the data anonymous to all database users. However, there is still possibility that this third party would leak out the name or bills of material to public. Therefore, few manufacturers are willing to share their data.

\section{Kay Assumptions}

Current circumstances do not only depend on platform or engineering, but also on the natural unwillingness on data sharing. Companies or organizations are more concerned with personal gains or losses during data sharing, instead of how data sharing can benefit them and the whole industry. It is understandable that companies or organizations are responsible of keeping their own data confidential. However, this is one of the most important factors that impedes a good data sharing atmosphere. To solve this problem, an engineering solution is not sufficient. A more powerful and effective administrative solution should come out, to help guide companies to a beneficial solution.

There are three major assumptions that this study is based on:

1. Companies or organizations are willing to share their data and improve the environmental performance;

2. Economic consideration is not the only threshold to consider during the selection on alternatives. Environmental impact also plays a significant role, and the product with better environmental performance could be more attractive;

3. Stakeholders are willing to pay more for environmentally friendly products.

Under these assumptions, this study provides a solution to do automatic LCI data transmission and LCA calculation.

\section{Framework and Mechanism}

In this study, by applying blockchain technology, BC-LCA can replace the traditional centralized structure and improve the efficiency in data transmission. In BC-LCA, all the data verifications and restrictions can be done by an arbitrary node, instead of a fixed "center" (Figure 3), thereby drastically improving the efficiency of data transmission. However, there's one thing that needs to be considered: every node should contain the rule to do restrictions and verifications. In other words, if a manufacturer decides to join in the BC-LCA, this manufacturer should act as a node with the full function to do the verifications and imply all the restrictions. Thus, BC-LCA requires a more meticulous design than traditional LCA framework. BC-LCA can overcome the limitation of centralized structure and improve the performance of classic LCA.

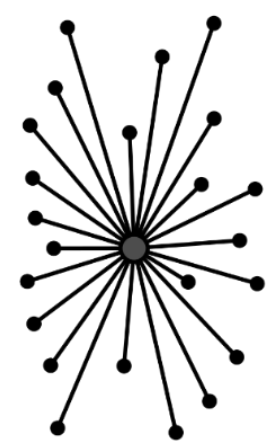

A

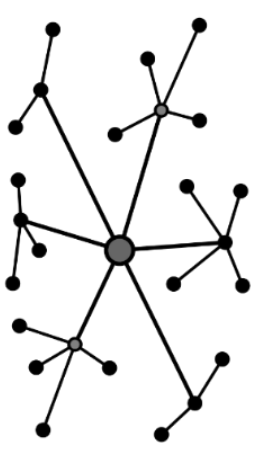

B

Figure 3. Graphical comparison of a centralized (A) and a decentralized (B) system. 
Based on the classic LCA framework and classic blockchain framework (Figure 1), the new blockchain based LCA network could have the full function in both LCA and blockchain. It could analysis the environmental impact of a product or process, and all the data transmission would go through the blockchain. The new blockchain-based LCA framework would also be more automatic and accurate.

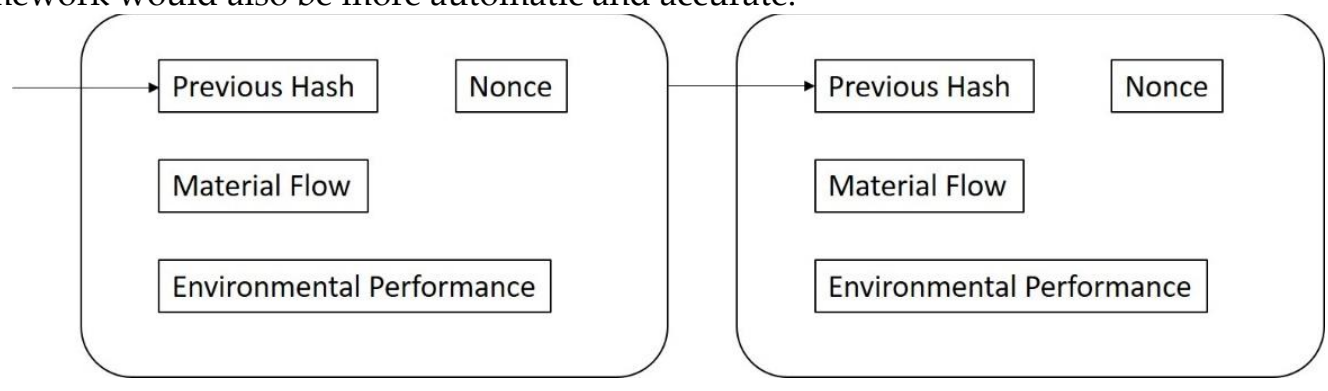

Figure 4. Framework of BC-LCA.

Figure 4 shows the framework of BC-LCA. In every block, "Previous Hash" and "Nonce" acts as linkage between blocks. "Material Flow" and "Environmental Performance" data would be stored in each block. In this case, during every time that a material flow occurs, this data would be broadcast into the BC-LCA network, and environmental performance of this material flow would be sent directly to the downstream node (which is the receiver). Each block has its capacity limitation, which means one block can store limited number of material flow data and environmental performance data. Thus, after one block is filled up, an arbitrary node would come up and "seal" the whole block and broadcast the block to the whole network.

The mechanism of BC-LCA is simple, but powerful. All information would be transferred via broadcast to the whole BC-LCA network. There are three types of nodes that participate in this information transfer: sending node, receiving node and transfer nodes. Sending node is the one who broadcast information into BC-LCA network, as well as the node who send out material flow. Receiving node is the node that receives the material flow. Transfer nodes are these nodes that only participate in information transfer but are not the sender nor receiver nodes.

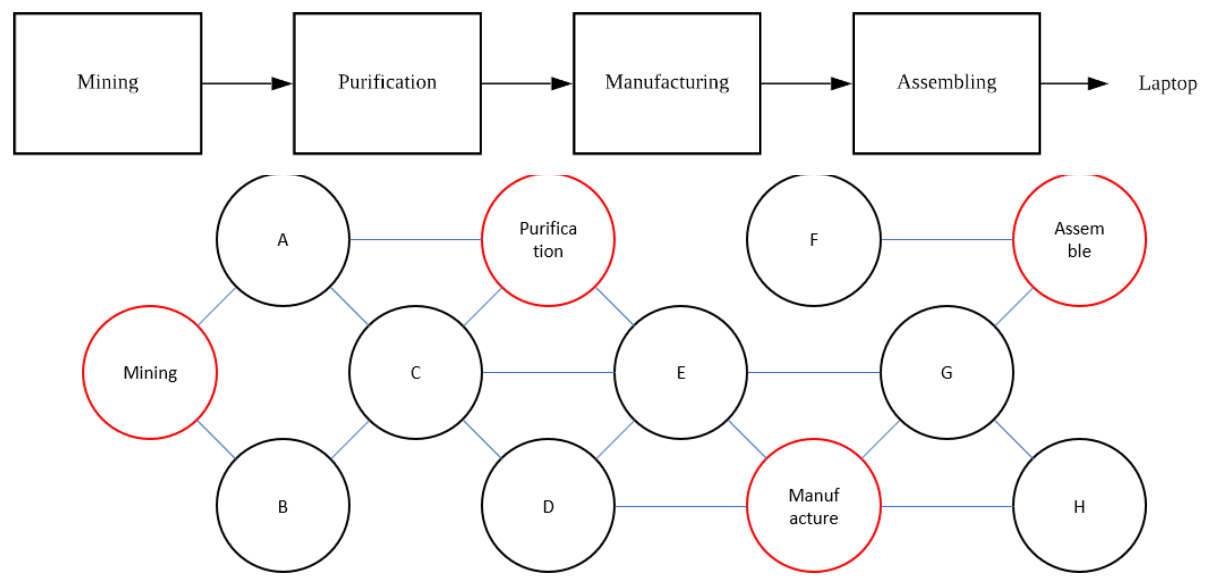

Figure 5. Decentralized LCA Network Sample.

Figure 5 shows an example of information transfer. Node Mining want to transfer 1 tons of Product A to Node Purification, which is defined as "material flow". Simultaneously, Node Mining would broadcast this information to the whole network with the following procedure:

1. Getting information ready: Node Mining will need to prepare the list of products (1 tons of Product A), and environmental impacts related to these products (environmental impacts of Product A); 
2. Notify Node A and Node B: The closest nodes of Node Mining are Node A and Node $\mathrm{C}$, which would be the aim of broadcasting. Here, Node Mining would send out the prepared information to Node A and Node B directly;

3. Node A would notify Node Purification and Node B would notify Node C: However, the closest nodes for Node $\mathrm{A}$ are Purification and $\mathrm{C}$ and the closest node of Node $\mathrm{B}$ is C. Therefore, Node A will send information to Node Purification and Node C, and Node B will also send information to Node C. It does not matter who will send Node $\mathrm{C}$ the information first, as finally both Node $\mathrm{C}$ and Node Purification gets the necessary prepared information;

4. Node Purification receive environmental impacts, all other nodes keep the record: After the prepared information received by Node Purification, Node A, Node B and Node $C$ would always keep the prepared information. Furthermore, the prepared information would keep spreading, until it is received in all the nodes in the BC-LCA network. Here, beside Node Mining and Node Purification, all other nodes would keep the prepared information for record and prevent cheating.

There would be bunch of information spreading in BC-LCA network at the same time. However, it is not necessary to worry if one information would be transferred duplicated between one node pair. Every time when a node receives an info, the node would check if this info already existed. Any node would only accept the information which is not received. When the last node in BC-LCA receive the information, the node would find no other node to send, then the whole broadcasting is over.

In BC-LCA, any node can perform the LCA calculation. At the time a node receives a material flow information, it would automatically calculate the environmental impact. Here, the update of products' environmental performance occurs every time this node receives material flow information. The calculation would be consisted of two parts: bills of material and LCI data. Both parts come from BC-LCA network and could be auto updated if the upstream nodes provide update information. This calculation could directly provide the results of product final environmental inventory table, and broadcast to the whole BC-LCA network.

Addition to the external-node data transition, there are three pre-processes that should be considered in the internal-node calculation:

1. Bills of material confirmation: The very first step before calculation, is to make sure the input data is valid. Here, a node needs to check whether the input bills of material table is legal, and whether each element of this table has appropriate format;

2. LCI dataset confirmation: The second step is to confirm the LCI dataset is up-to-date and contain all the information to calculate. Each node should have multiple versions of LCI dataset, including the dataset directly from upstream nodes, and the dataset for recording only. Before any calculation, a node should search for and sort out appropriate LCI dataset;

3. Unit consistent inspection: After confirming bills of material and LCI dataset, the next step should be checking the unit consistence. Different unit could lead significant impact to environmental performance factors. The unit in bills of material should match with the unit in the LCI dataset.

After these pre-processes, a node could perform a matrix multiplication [18], to figure out environmental impact results of one product. Multiple products need multiple calculations, with all 3 pre-process steps for each calculation. When a node finishes an environmental impact analysis for one product, this node can then broadcast the results to the whole BC-LCA network. Then, all downstream nodes, which may be impacted by this analysis, could update their related products. The whole process, starting with the first node who change its product environmental impact data, until the last node finishes updating, is called cascading.

\section{Benefits of BC-LCA}




\subsection{Data Availability}

To make the accurate environmental inventory data available for a specific product, the first step is to acquire the accurate data on every item in this product's bills of material, which means the upstream nodes (i.e., suppliers) should provide accurate information for their products. The easiest way to let the supplier provide the environmental inventory data for their products, is bringing the supplier into the same blockchain network. In this case, while material goes from upstream to downstream, the environmental inventory data should follow the same direction. This is the meaning of blockchain based LCA network: digitization all the material flow and providing accurate data to all the nodes.

\subsection{Data Privacy}

Benefited by the cryptocurrency, blockchain technology has already has a practical enciphering algorithm: Security Hash Algorithm (SHA), published by National Institute of Standards and Technology (NIST) as a U.S. Federal Information Processing Standard (FIPS) [19]. This method can promise that enciphered private information can only be read or edited by the user who has the key, with no exception. Key is a string with the information to decipher user's private data. Even the enciphered data owner cannot read the enciphered data without the key. By doing this, user's privacy could be protected, and they could be more willing to share their environmental inventory data of their products to the downstream users.

Here recall Figure 5 as example. When Node Mining sends 1 ton of Product A to Node Purification, a Key, which contain the information to decipher, would be sent from Node Mining to Node Purification. Then the transition of material happens, and transition information ( 1 ton of Product A and the environmental impact of 1 ton of Product A) would be broadcasted to the whole network. However, during this step, the product list ( 1 ton of Product A) is enciphered, but the environmental inventory information (the environmental impact of 1 ton of Product A) is open to public. The sender and receiver are also anonymous, which means all other nodes (except Node Mining and Node Purification) can only see that Node $X$ send Node $Y$ some unknown product, together with a certain known amount of environmental impact. And Node Purification, who has the Key from Node Mining, can decipher the broadcasting information, and know that this transmission includes 1 ton of Product A, and calculate out the environmental impact of 1 unit of Product A.

\subsection{Cheating Prevention}

In the long term, all nodes should have a balanced input and output environmental impact, even consider the inventory of storage. With BC-LCA, preventing cheating becomes easy: any node can calculate the input and the output environmental impact and therefore can tell whether there any cheating has occurred. Just like Bitcoin network, a user can know the budget in his/her account, any BC-LCA user is able to know what's the accumulative environmental impact in its node. Because every transaction in BC-LCA has a timestamp, it would be easy to track the growth/decay rate of accumulative environmental impact and use an algorithm to judge if that node is cheating or not.

\section{Case Study based on an Industrial Supply Chain}

Willems provides some simplified supply chain data [20], which could be used to demonstrate the application of BC-LCA. Here, an industrial organic chemical supply chain is selected, whose picture is shows as Figure 6. 


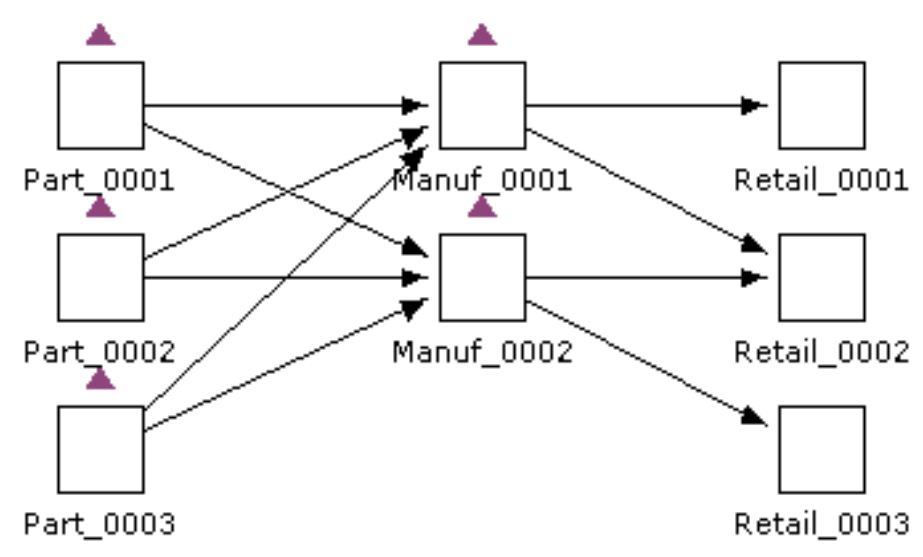

Figure 6. Sample supply chain picture [20].

This supply chain includes 3 procure stages, 2 manufacture stages, no transportation stage and 3 retail stages. In total, there are 8 nodes with 10 edges. This supply chain is comparatively simple, but it could show the difference with and without BC-LCA application. Here, a Python based BC-LCA is developed for simulation.

Due to the lack of material flow information in the supply chain sample, five assumptions are made:

1. Manuf_0001 and Manuf_0002 share the same manufacturing recipe;

2. Carbon footprint is calculated using US-EEIO based on cost data provided;

3. All nodes locate in California;

4. Only carbon emission is considered;

5. Transactions (material flow) between any two connected nodes happens once a month;

6. The whole model runs from Jan-2018 until July-2020;

In this case study, five scenarios are considered:

0. No node joins BC-LCA

1. Only Manuf_0002 joins BC-LCA

a. The percentage of clean energy using in manufacturing is increasing [21-23]

b. Due to the procedure upgrade, material waste is decreasing

c. The energy consumption for supplement (light, A/C, etc.) varies seasonally

2. Part_0001, Part_0002, Part_0003 and Manuf_0002 join BC-LCA

a. The energy consumption to fetching raw material is decreasing

b. The percentage of clean energy used in manufacturing is increasing due to assumption 3

c. Due to the procedure upgrade, material waste is decreasing

d. The energy consumption for supplement (light, A/C, etc.) varies seasonally

3. Only Manuf_0002 joins BC-LCA

a. The energy consumption is increasing due to the aging of equipment

b. The energy consumption for supplement (light, A/C, etc.) varies seasonally

4. Part_0001, Part_0002, Part_0003 and Manuf_0002 join BC-LCA

a. The energy consumption to fetching raw material is decreasing

b. The energy consumption is increasing due to the aging of equipment

c. The energy consumption for supplement (light, A/C, etc.) varies seasonally 
Because Manuf_0001 never joined BC-LCA, instead, Manuf_0001 always use classic LCA, and the results stay constant over years. The result of Manuf_0001 could be used as a baseline to compare with Manuf_0002, which contains more conditions, and updated monthly. Based on these scenarios, results are shown as below:

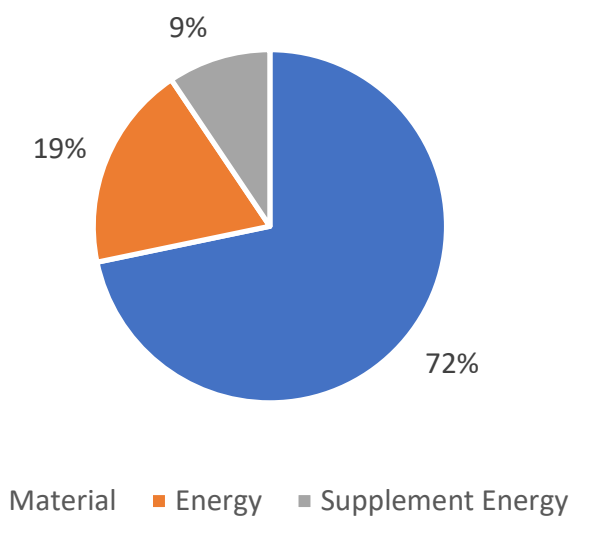

Figure 7. Carbon Emission of Final Product under Scenario 0.

Figure 7 shows the carbon emission of final product under Scenario 0, which considered no node joining the BC-LCA. All carbon emission data are calculated from US-EEIO v2.0 [24], where a factor is provided, so that the stage cost of a node can be directly convert to carbon footprint. Scenario 0 is a baseline, where carbon emission would not change, and all results depend on the manufacturing recipe.

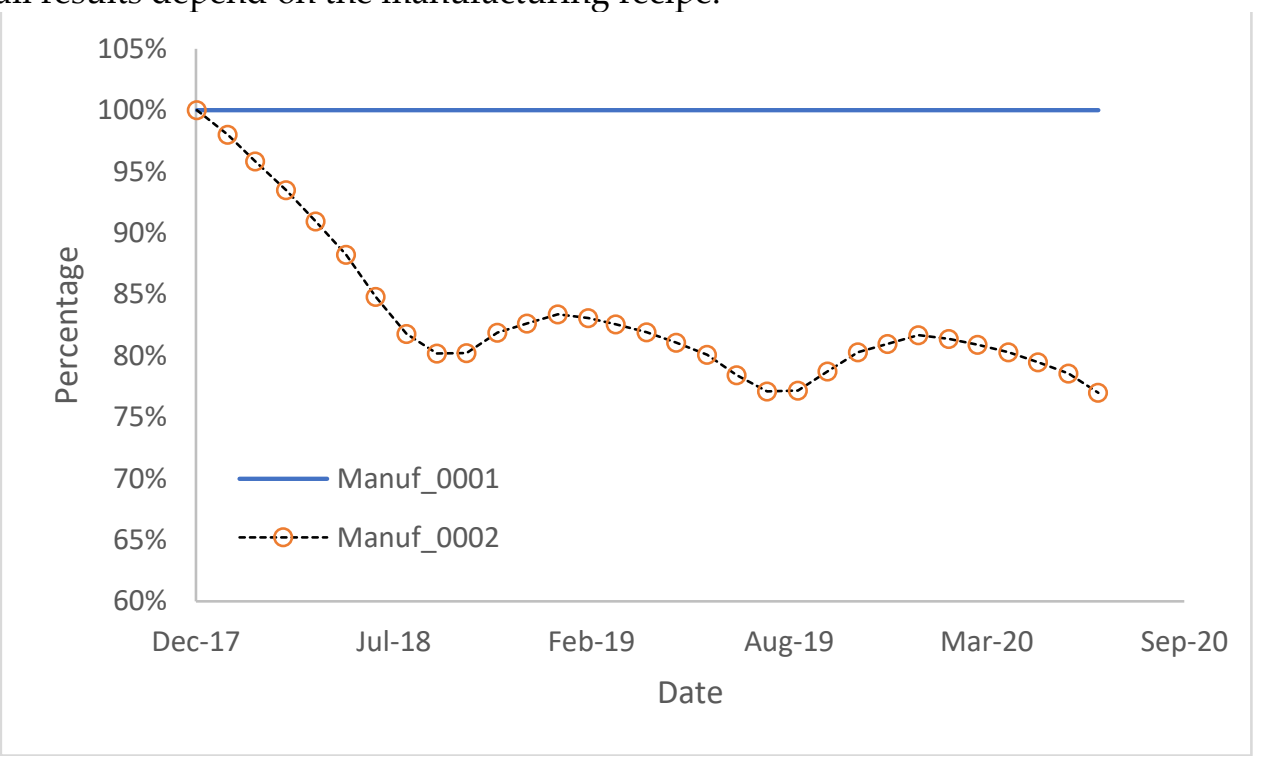

Figure 8. Carbon Emission Compare Percentage of Final Product under Scenario 1.

Figure 8 shows the carbon emission under Scenario 1. It is obvious that due to condition $1 \mathrm{a}$ and condition $1 \mathrm{~b}$, the overall trend of carbon emission is decreasing. Some variance in this curve is due to condition 1c, which means in every winter, Manuf_0002 would need more energy to keep room temperature. 


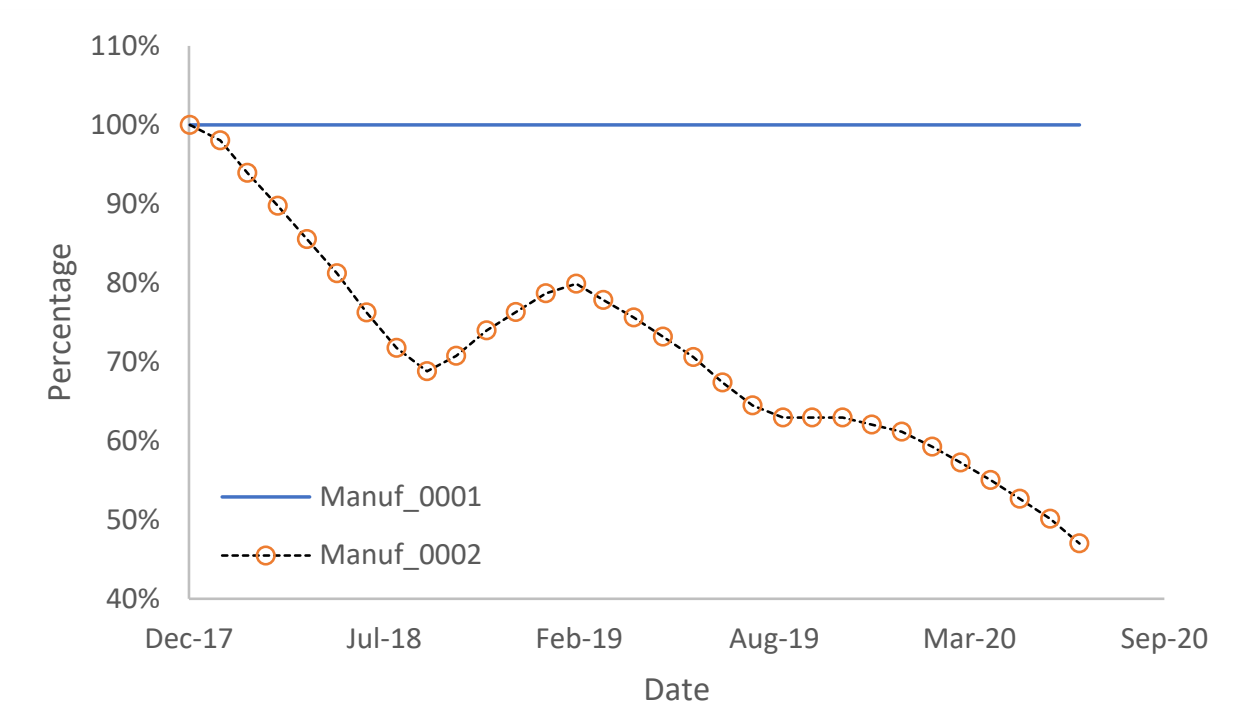

Figure 9. Carbon Emission Compare Percentage of Final Product under Scenario 2.

For Scenario 2, BC-LCA performs more accurate and variable results. In Figure 9, when more nodes join in BC-LCA, the overall trend of decarbonization is more obvious, which depends on condition 2.a.

With the comparison of Figure 8 and Figure 9, which represent the carbon emission variance when only 1 node joins BC-LCA and when 4 nodes join BC-LCA, it would be safe to say that with more node in one supply chain join BC-LCA, the results of environmental impact would be more and more accurate. The reaction to periodical waving and long-term trend is both shown in Scenario 1 and Scenario 2.

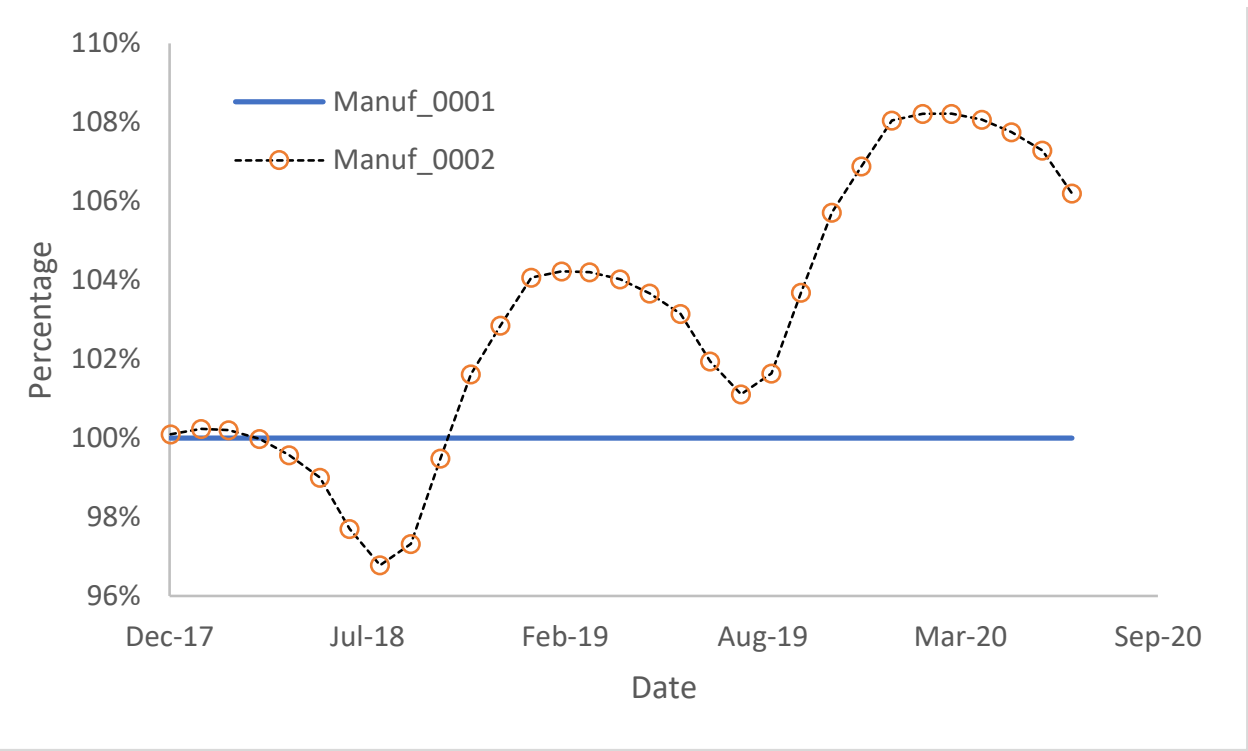

Figure 10. Carbon Emission Compare Percentage of Final Product under Scenario 3.

However, the decreasing of overall carbon emission is due to the scenario assumptions. The results from BC-LCA could only be more accurate, but not necessarily more environmentally friendly, than traditional LCA. Figure 10 shows the carbon emission results under Scenario 3, where the overall trend of increasing carbon emissions is mostly due to condition 3a. 


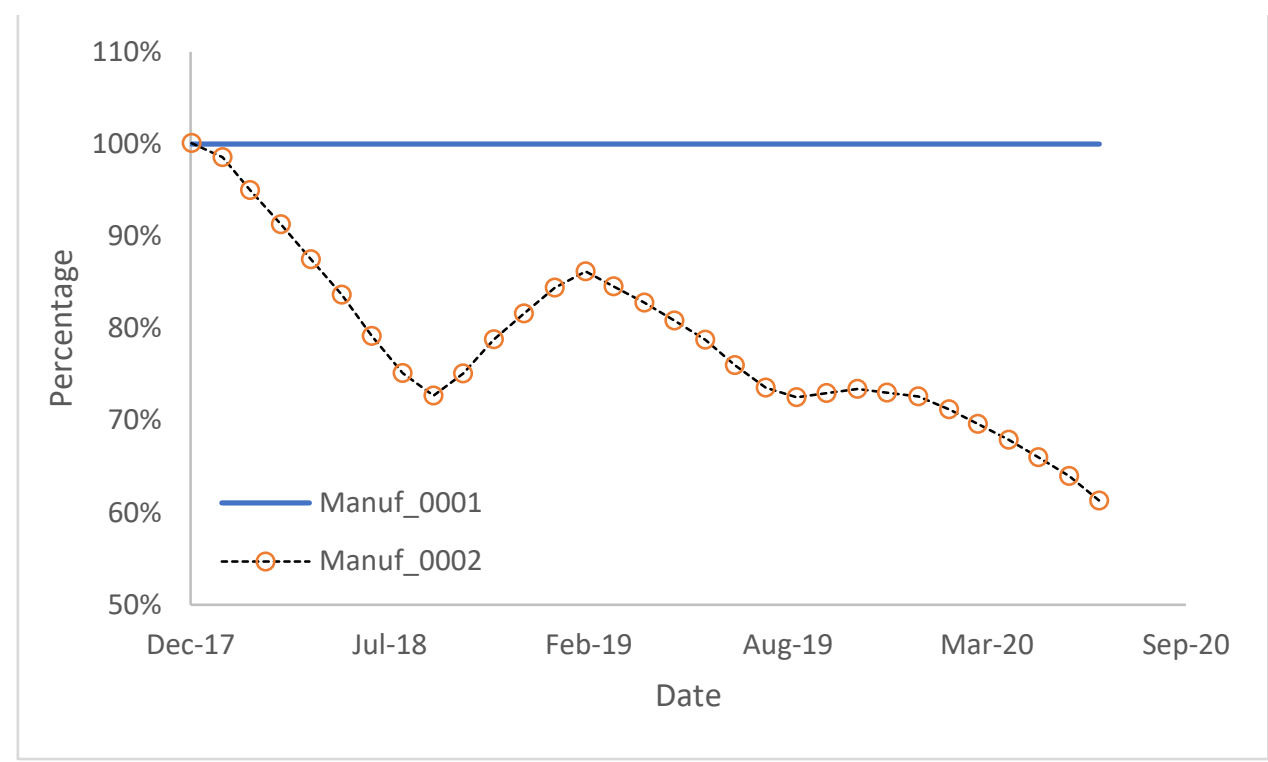

Figure 11. Carbon Emission Compare Percentage of Final Product under Scenario 4.

Figure 11 shows that, under Scenario 4, even though the energy consumption in Manuf_0002 is increasing due to the aging of equipment, the overall carbon emission is still decreasing.

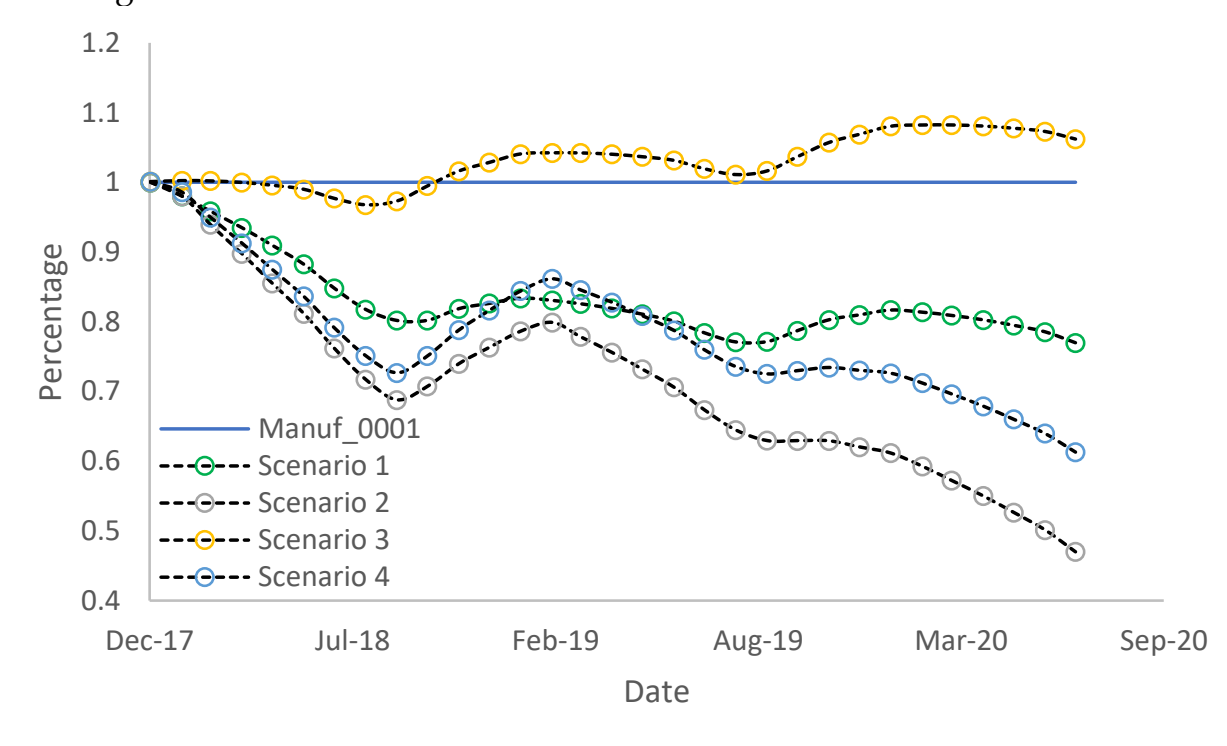

Figure 12. Carbon Emission Comparison of All Scenarios.

Based on these scenarios, some comparisons are made (Figure 12). The analysis results show that even this case study implies a simple supply chain, the difference between BC-LCA and classic LCA is significant. Carbon emission results are more accurate and specific. Manuf_0001 carbon emission could be seen as an average, which is good for general analysis. When Manuf_0002 join the BC-LCA (Scenario 1 and 3), it is obvious to see how energy consumption, material waste and supplement energy consumption affect carbon emission. When Part_0001, Part_0002 and Part_0003 also joined in BC-LCA (Scenario 2 and 4), which means the whole supply chain join BC-LCA, more factors are considered, so more variances could be observed.

During the whole case study, all data transmissions are under blockchain rule. As mentioned before, bills of materials would be enciphered, but total environmental impact of one material flow would be open to public. The strength of SHA-256 is much higher than classic security method like username and password [25]. 
Table 1. Sample Transmission Data without Encipher

\begin{tabular}{ccccccc}
\hline Date & Sender & Receiver & Quantity & Unit & Material & Carbon \\
\hline Jan-18 & Part_0002 & Manuf_0002 & 12 & Dollar & Material 1 & 76.31688 \\
Feb-18 & Part_0002 & Manuf_0002 & 12 & Dollar & Material 1 & 76.31688 \\
Mar-18 & Part_0002 & Manuf_0002 & 12 & Dollar & Material 1 & 76.31688 \\
Apr-18 & Part_0002 & Manuf_0002 & 12 & Dollar & Material 1 & 76.31688 \\
May-18 & Part_0002 & Manuf_0002 & 12 & Dollar & Material 1 & 76.31688 \\
Jun-18 & Part_0002 & Manuf_0002 & 12 & Dollar & Material 1 & 76.31688 \\
Jul-18 & Part_0002 & Manuf_0002 & 12 & Dollar & Material 1 & 76.31688 \\
\hline
\end{tabular}

Table 2. Sample Transmission Data with Encipher

\begin{tabular}{ccccccc}
\hline Date & Sender & Receiver & Quantity & Unit & Material & Carbon \\
\hline Jan-18 & Bgrws2dNbee6q6aIaOsf & FzYNXJMtz0UbrAyDvrij & O4UKAYzZuA & Dollar & YpMJUyhQEq & 76.31688 \\
Feb-18 & Bgrws2dNbee6q6aIaOsf & FzYNXJMtz0UbrAyDvrij & TOx3cUVgxl & Dollar & zVjKPSjWVB & 76.31688 \\
Mar-18 & Bgrws2dNbee6q6aIaOsf & FzYNXJMtz0UbrAyDvrij & rFxnZB7ucf & Dollar & osIg5wtyXP & 76.31688 \\
Apr-18 & Bgrws2dNbee6q6aIaOsf & FzYNXJMtz0UbrAyDvrij & tMSkZ1BL7x & Dollar & 2 2qWqlOpiiJ & 76.31688 \\
May-18 & Bgrws2dNbee6q6aIaOsf & FzYNXJMtz0UbrAyDvrij & n7XXrxdorE & Dollar & QWWMvygVbs & 76.31688 \\
Jun-18 & Bgrws2dNbee6q6aIaOsf & FzYNXJMtz0UbrAyDvrij & 5VXDudTF7c & Dollar & lu0KP0eM6H & 76.31688 \\
Jul-18 & Bgrws2dNbee6q6aIaOsf & FzYNXJMtz0UbrAyDvrij & qzNTN8oqs8 & Dollar & Ev1G091PC2 & 76.31688 \\
\hline
\end{tabular}

Table 1 and Table 2 shows the result of encipher, which is manually exported and formatted from BC-LCA, based on Scenario 4. Generally, when BC-LCA network is running, the result of encipher would be stay in catcher, and not that easy to observe. The encipher and decipher operation would be run automatically. Compare Table 1 and Table 2 , the name of sender and receiver is constant, though anonymous. The material name after enciphering is dynamic, which means every transaction has its unique Key to decipher, to enhance data privacy.

Another test is also performed to exam the ability of cheating prevention, based on Scenario 4. Manuf_0002 is manually set to cheat, which it claims that its products have $10 \%$ lower carbon footprint than they actually have. In this case, some carbon footprint would be accumulated in Manuf_0002. And a threshold is set, to make sure that any node with higher accumulation carbon footprint high than the threshold, would be warned and kicked out.

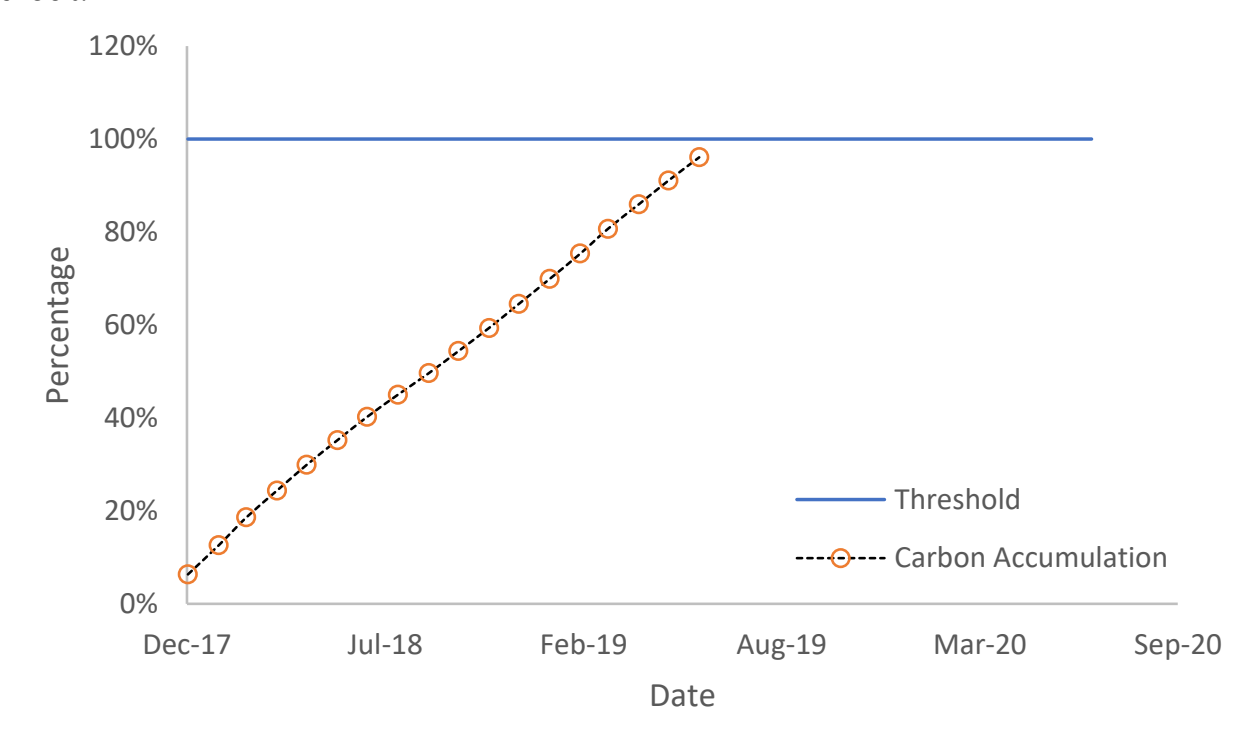

Figure 13. Carbon Accumulation Percentage when Cheating. 
Figure 13 shows the carbon accumulation percentage compared to threshold. The carbon footprint is always accumulated, and when it reaches the threshold at Jul-2019, Manuf_0002 is warned and kicked out of BC-LCA network. The threshold could be modified according to actual requirement.

The whole case study is programmed with Python, where a simple version of BCLCA is developed, with limited nodes and transactions. However, this framework could be expanded to a supply chain across multiple companies, or multiple departments within one big company.

\section{Conclusions and Future Work}

BC-LCA is the combination of blockchain technology and life cycle assessment, which could significantly improve the availability, privacy, accuracy, and timeliness of LCI data, with less manual operation and time cost. The framework and mechanism of BC-LCA are both designed based on blockchain and modified according to life cycle assessment features. The whole network can automatically calculate environmental impact, transfer data, back up data and prevent cheating.

Next step of this research may include an algorithm and data structure design of BCLCA, as well as a practical demonstration to test the time and hardware cost when BCLCA is implemented.

\section{Author Contributions}

Conceptualization, Xuda Lin and Xing Li; Formal analysis, Xuda Lin; Methodology, Xuda Lin and Xing Li; Project administration, Fu Zhao; Software, Xuda Lin; Validation, Fu Zhao; Writing - original draft, Xuda Lin; Writing - review \& editing, Xuda Lin, Xing Li, Sameer Kulkarni and Fu Zhao.

\section{References}

1. Luthra, S.; Mangla, S.K. When Strategies Matter: Adoption of Sustainable Supply Chain Management Practices in an Emerging Economy's Context. Resources, Conservation E Recycling 2018 138,194-206.

2. Balaguera, A.; Carvajal, G.I.; Albertí, J. Life Cycle Assessment of Road Construction Alternative Materials: A Literature Review. Resources, Conservation E Recycling 2018 132, 37-48.

3. Levasseur, A.; Cavalett, O.; Fuglestvedt, J.S. Enhancing Life Cycle Impact Assessment from Climate Science: Review of Recent Findings and Recommendations for Application to LCA. Ecological Indicators 2016 71,163-174.

4. Nakamoto, S. Bitcoin: A peer-to-peer electronic cash system. Manubot 2019.

5. Saberi, S.; Kouhizadeh, M.; Sarkis, J. and Shen, L. Blockchain Technology and Its Relationships to Sustainable Supply Chain Management. International Journal of Production Research 2019 57.7, 2117-135.

6. Min, H. Blockchain Technology for Enhancing Supply Chain Resilience. Business Horizons 2019 62.1, 35-45.

7. Chang, S.K. Data Structures and Algorithms. 1st ed.; World Scientific: Hong Kong, China, 2003.

8. Smetana, S.; Seebold, C.; and Heinz, V. Neural network, blockchain, and modular complex system: The evolution of cyberphysical systems for material flow analysis and life cycle assessment. Resources, Conservation E Recycling 2018 133, $229-230$.

9. Zhang, A.; Zhong, R.; Farooque, M. Blockchain-based life cycle assessment: An implementation framework and system architecture, Resources, Conservation \& Recycling 2020 152, 104512.

10. Farooque, M.; Jain, V.; Zhang, A. Fuzzy DEMATEL analysis of barriers to Blockchain-based life cycle assessment in China. Computer E Industrial Engineering 2020 147: 106684.

11. Teh, D.; Khan, T.; Corbitt, B. Sustainability strategy and blockchain-enabled life cycle assessment: a focus on materials industry. Environment Systems and Decisions 2020 40,605-622.

12. Rolinck, M.; Gellrich, S.; Bode, C. A Concept for Blockchain-Based LCA and its Application in the Context of Aircraft MRO. Procedia CIRP 2021 18,394-399.

13. Fang, H.; Jiang, D.; Yang, T.; Fang, L. Network Evolution Model for Supply Chain with Manufactures as the Core. PLoS ONE 2018 13.1, E0191180

14. Hospido, A.; Davis, J.; Berlin, J.; and Sonesson, U. A Review of Methodological Issues Affecting LCA of Novel Food Products. The International Journal of Life Cycle Assessment 2010 15.1, 44-52.

15. Mcelheran, K. Decentralization versus Centralization in IT Governance. Communications of the ACM 2012 55.11, 28-30.

16. Minas, R.; Wright, S.; and Berkel, R.V. Decentralization and Centralization. International Journal of Sociology and Social Policy 2012 32.5/6, 286-98. 
17. Wernet, G.; Bauer, C.; Steubing, B. The ecoinvent database version 3 (part I): overview and methodology. The International Journal of Life Cycle Assessment. 2016 21, 1218-1230.

18. Heijungs, R., Suh, S., The computational structure of life cycle assessment, 1st ed.; Kluwer Academic Publishers, 2002.

19. Stevens, M.; Bursztein, E.; Karpman, P.; Albertini, A.; \& Markov, Y. The First Collision for Full SHA-1. Advances in Cryptology CRYPTO 2017, 570-596.

20. Willems, S. Data Set - Real-Word Multiechelon Supply Chains Used for Inventory Optimization. Manufacturing \& Servide Operations Management 2008 10(1), 19-23.

21. U.S. EIA. California State Energy Profile, 2019

22. U.S. EIA. California State Energy Profile, 2020

23. U.S. EIA. California State Energy Profile, 2021

24. Yang, Y.; Ingwersen, W.W.; Hawkins, T.R.; Srocka, M.; \& Meyer, D.E. USEEIO: A new and transparent United States environmentally-extended input-output model. Journal of cleaner production 2017 158, 308-318

25. Velmurugadass, P.; Dhanasekaran, S.; Anand, S.S. Enhancing Blockchain security in cloud computing with IoT environment using ECIES and cryptography hash algorithm. Materials Today: Proceedings 2021 37,2653-2659.Velmurugadass, P., Dhanasekaran, S., Anand, S. S.. “Enhancing Blockchain security in cloud computing with IoT environment using ECIES and cryptography hash algorithm". (2021) 37:2653-2659. 